\title{
Facilitators and Barriers to Implementation of the Pharmacy DOTS Initiative in Selected Project Sites in the Philippines
}

\author{
Jonathan P. Guevarra, RN, RM, MAN, ${ }^{1,2}$ Carl Abelardo T. Antonio, MD, MPH, ${ }^{3,4}$ Amiel Nazer C. Bermudez, MD, MPH, ${ }^{5,6}$ \\ Kim L. Cochon, MSPH, ${ }^{7}$ Azar G. Agbon, ${ }^{8}$ Michelle D. Avelino, ${ }^{8}$ Jorel A. Manalo, MPH, ${ }^{9}$ \\ Diana Dalisay A. Orolfo, ${ }^{8}$ Eden C. Mendoza, MD, $\mathrm{MPH}^{10}$ and Ronald Allan M. Fabella, $\mathrm{MD}^{10}$
Department of Health Promotion and Education, College of Public Health, University of the Philippines Manila, Manila, Philippines
${ }^{2}$ College of Development Communication, University of the Philippines Los Baños, Laguna, Philippines
${ }^{4}$ Department of Applied Social Sciences, The Hong Kong Polytechnic University, Kowloon, Hong Kong
${ }^{5}$ Department of Epidemiology and Biostatistics, College of Public Health, University of the Philippines Manila, Manila, Philippines
${ }^{6}$ Department of Epidemiology, School of Public Health, Brown University, Rhode Island, United States of America
${ }^{7} J C$ School of Public Health and Primary Care, Faculty of Medicine, The Chinese University of Hong Kong, Hong Kong SAR
${ }^{8}$ College of Public Health, University of the Philippines Manila, Manila, Philippines
${ }^{9}$ Human Resource Development Office, University of the Philippines Manila, Manila, Philippines
${ }^{10}$ Philippine Business for Social Progress, Manila, Philippines \\ ${ }^{3}$ Department of Health Policy and Administration, College of Public Health, University of the Philippines Manila, Manila, Philippines
}

\begin{abstract}
Objectives. The Pharmacy DOTS Initiative (PDI) was relaunched on a larger scale in 2014 through the Innovations and Multi-Sectoral Partnerships to Achieve Control of Tuberculosis (IMPACT) project. This paper aimed to assess the PDI program through IMPACT by identifying the facilitating and hindering factors in its implementation. The identified factors are classified as to the affected stakeholders or processes.
\end{abstract}

Methods. Semi-structured interviews were conducted with the PDI Program Manager and four NTP coordinators from selected project sites. Thematic analysis was done to determine the recurring facilitating and hindering factors as identified by the key informants.

Results. Facilitating factors identified include cooperation of the stakeholders, capability-building and a good referral system. The barriers to the implementation were grouped into patient-related, pharmacy-related, health centerrelated, program-related as well as external factors.

Conclusion. The referral system created through PDI facilitated the flow of referrals starting from the pharmacy. This enabled presumptive patients to have access to health facilities for TB. Hindering factors contributed to the inability of the engaged pharmacies to sustain their consistency and commitment in conducting the PDI interventions.

Key Words: barriers, facilitators, tuberculosis, directly observed therapy, program evaluation, pharmacy

\section{INTRODUCTION}

Corresponding author: Jonathan P. Guevarra, RN, RM, MAN Department of Health Promotion and Education College of Public Health

University of the Philippines, Manila

Room 101 Mezzanine, Lara Hall,

625 Pedro Gil Street, Ermita, Manila 1000, Philippines

Email: jpguevarra2@up.edu.ph
The Philippines has been included in the list of 30 countries with the highest burden of tuberculosis (TB) cases worldwide with a reported incidence of 322 per 100 , 000 population (95\% uncertainty interval (UI): $277-370$ per 100, 000). ${ }^{1}$ The Directly Observed Treatment Shortcourse chemotherapy (DOTS) strategy is being used across the country for TB treatment. ${ }^{2}$ The problem with TB treatment has worsened due to the emergence of drugresistant strains. ${ }^{3}$ This is partly facilitated by the practice of self-medication by Filipinos with symptoms of TB. ${ }^{4}$ Selfmedication complicates treatment because the usual regimen 
for TB is not sufficient to eradicate resistant strains. ${ }^{1}$ This practice places pharmacists in an important position in the prevention, detection and management of presumptive and confirmed TB cases $^{5}$, which led to the conceptualization of the Pharmacy DOTS Initiative (PDI).

The PDI was formed in 2004 by the Philippine Tuberculosis Initiatives for Private Sector (PhilTIPS) with support from the United States Agency for International Development (USAID). PDI was relaunched in 2010 by the Linking Initiatives and Networking to Control Tuberculosis (TB LINC) and in 2012 by the Philippine Pharmacists Association ( $\mathrm{PPhA})$. Under this project, pharmacies and its personnel were expected to contribute to the prevention of TB self-medication through appropriate education and referral, institution of behavior change among the pharmacy personnel towards participation in the National Tuberculosis Control Program (NTP) and strengthening and implementation of national and local policies supporting TB DOTS. The pilot programs contributed to the case detection and cure rate in the implementation sites but its impact remained low due to the small number of participating pharmacies. To introduce the program in a large-scale set-up, the Philippine Business for Social Progress (PBSP) awarded a sub-grant to PPhA through the Innovations and MultiSectoral Partnerships to Achieve Control of Tuberculosis (IMPACT) project funded by the USAID for the period from January 2014 to September 2016. ${ }^{6}$

This most recent iteration of the PDI involved a total of 7,138 pharmacies in 33 implementation sites across the country, which were expected to deliver three main services: (a) provision of information and patient counselling to clients of the drugstores; (b) implementation of the "No prescription, No dispensing" policy for anti-TB drugs; and (c) referral of identified clients to DOTS facilities. Participating pharmacies were expected to record the number of clients to whom the "No prescription, no dispensing" policy and referrals were made. DOTS facilities were required to document the number of referrals that came from drugstores. PDI aimed to increase case detection of TB and increase the number of participating pharmacies to ensure sustainability of the program.

The program was implemented under the direct supervision of the PDI Program Manager who coordinated with the stakeholders and the rest of the PDI project team. Aside from the PDI project team, local government units (LGUs) especially DOTS facilities and the local NTP coordinators of the evaluation sites were involved in the project's implementation. LGUs and local NTP coordinators were given an orientation regarding the interventions to be implemented under PDI as part of the mapping and engaging of pharmacies. The Program Manager coordinated with the Chapter Presidents of $\mathrm{PPhA}$ in the PDI implementation sites, deans of the pharmacy schools within the area, and the Area Field Coordinators. The Program Manager also served as the official representative of the sub-grantee and maintained communication among the stakeholders and other relevant parties. NTP Coordinators included staff from the $\mathrm{DOH}$ responsible for the implementation of NTP in their assigned sites.

The implementation of PDI in the selected sites was intended to demonstrate the feasibility and scalability of the intervention as a support measure within the broader strategy and framework of TB prevention and control in the country. Thus, IMPACT-PBSP commissioned an evaluation of PDI to identify factors that may have contributed positively to or hindered the attainment of the program outcomes, which was reported in two previously published papers, ${ }^{7,8}$ to inform future iterations of the intervention. This paper aimed to identify facilitating and hindering factors on the implementation of PDI in selected project sites. The information can contribute to the improvement of PDI for a more sustainable implementation of the program as well as to maximize the program's impact on the TB situation in the country.

\section{MATERIALS AND METHODS}

There were five evaluation sites included in the study as identified by IMPACT; namely, Caloocan City; San Carlos, Pangasinan; Batangas City, Batangas; Dipolog City, Zambonaga del Norte; and Palo, Leyte. One evaluation site was selected from each city or province of the IMPACT regions. Four NTP coordinators from the evaluation sites, as well as the PPhA PDI Program Manager, were individually interviewed. The NTP Coordinator for one of the evaluation sites declined to participate in the interview because the informant was not yet part of the NTP when the PDI was implemented and rolled out in the said evaluation site, and no suitable replacement was identified in the area.

A semi-structured interview was conducted by two members of the evaluation team using a standard interview guide. The interview focused on establishing the role of the interviewee in the PDI implementation, opinions on the activities implemented under the PDI, and views on the acceptability and effectiveness of the technical assistance package of PDI. Informants were also asked about their insights on the facilitating and hindering factors that may have influenced the implementation of the PDI. The interview was audio recorded with the consent of the interviewees. Thematic analysis ${ }^{9}$ was done by the evaluation team after the transcription, translation, and coding of all interviews. Results were validated internally through discussion among the evaluation team members, as well as cross-checking of identified themes with field interviewers.

\section{RESULTS}

\section{Role in the implementation of the PDI}

Interviews with NTP coordinators revealed that their roles and responsibilities in the PDI implementation were 
not clearly defined and varied across the different sites. Two of the four NTP coordinators identified recruitment, orientation, and capacity-building sessions of PDI program for their involvement as a resource person or a supervisor. NTP coordinators from other areas identified accepting pharmacy referrals and providing TB treatment in their health facilities as their roles under PDI.

\section{Activities under the PDI}

The NTP coordinators outlined three major planned activities under PDI, namely: (1) engagement of private pharmacies, (2) referral of patients to health centers instead of dispensing anti-TB drugs, and (3) provision of incentives to pharmacies that actively refer TB patients. The incentives are available through the Provincial MultiSectoral Alliance, which is a collaboration of the provincial government and non-government organizations established with assistance from the USAID to expand both public and private sector participation to the NTP. The NTP Coordinators and the PDI Program Manager stated that all planned activities of PDI were implemented.

The PDI Program Manager was involved with the project from its conceptualization by PhilTIPS in 2004 until its reimplementation by TB LINC in 2010. The program manager created the program proposal that was submitted by PPhA to PBSP, and was later on assigned to oversee the project upon approval of the grant.

\section{Acceptability and Effectiveness of the Technical Assistance Package for PDI}

The technical assistance package for PDI was found to be helpful in the implementation of PDI in the evaluation sites. Drugstores refused dispensing anti-TB medications without prescription and referred identified patients to DOTS facilities. It improved the TB detection rate in one of the evaluation sites. Overall, the technical assistance package enabled the detection of new cases and provision of knowledge and skills to the LGU staff who were assigned to handle TB medicines. However, the use of the technical assistance package of PDI varied across drugstores and evaluation sites. The acceptability and effectiveness of the technical assistance package relied on the commitment of the drugstore and pharmacist to the program.

\section{Referral System during PDI Implementation}

The key informants stated that the referral system implemented under PDI was helpful in tracing and treating TB cases. Through the interview, the general flow of the TB referral system used in PDI was summarized (Figure 1). Patients who presented with cough of at least 2 weeks are referred to the City Health Office $(\mathrm{CHO})$ by the engaged pharmacies using the referral slips provided by the PDI program. The referral slips are collected in the $\mathrm{CHO}$ and the patients undergo preliminary assessment, including basic laboratory testing and procedures. After the

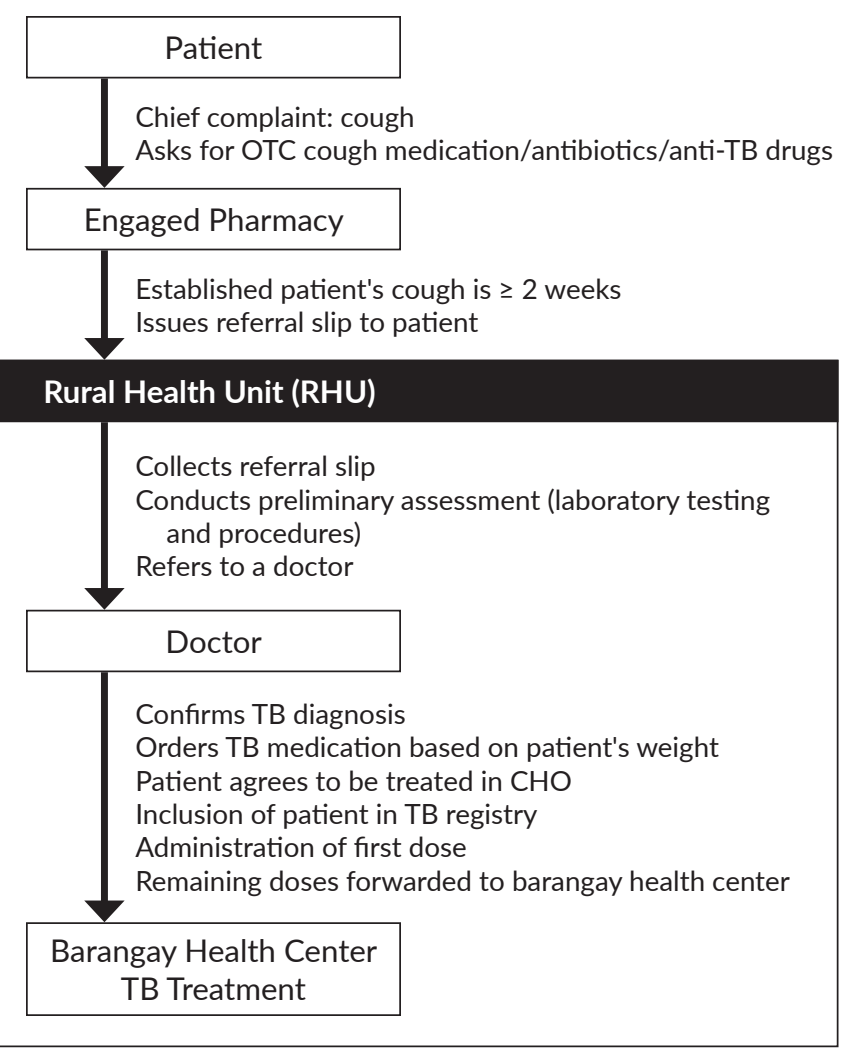

Figure 1. Referral system followed during the PDI implementation.

preliminary assessment, the patient is referred to a physician for consultation. A confirmed diagnosis of TB warrants a prescription of the appropriate dosage of $\mathrm{TB}$ medication. With this confirmed diagnosis, the patient must comply and agree to be treated in the $\mathrm{CHO}$ before being included in the TB registry. The first dose of anti-TB drugs is then given. The remaining doses of $\mathrm{TB}$ medication are forwarded to the respective barangay health center.

\section{Facilitating Factors in the PDI Implementation}

The primary facilitating factor in the implementation of PDI was the acceptance and cooperation of the stakeholders. The program was successfully implemented for a long period due to the commitment of the engaged pharmacies who believed in the program's advocacy and objectives. Through the PDI program, pharmacy personnel such as the pharmacists and pharmacy assistants were equipped with the knowledge and skills important in identifying and referring patients to DOTS facilities. The involvement of the health center through the referral system proved to be a vital factor in achieving the program objectives.

The establishment of the referral system created a link between the pharmacies and the health center. This facilitated the achievement of the program's goals. The flow of the referral system was facilitated by the acceptance and coordination of the public and private stakeholders. The government sector 
was engaged through courtesy calls conduct with the City or Provincial Health Officer and NTP Coordinators.

The personal connections of the PDI Program Manager facilitated the agreement of private sector stakeholders to collaborate under the program. Furthermore, the existing good relationship between the program manager and the pharmacist in the private corporation facilitated the commitment of the pharmacy chain company to PDI. The participation of big pharmacy chains contributed a significant number of referrals throughout the program implementation.

\section{Barriers to the PDI Implementation}

We identified barriers to implementation involving stakeholders or processes related to PDI into four clusters (Figure 2). NTP coordinators associated social stigma with $\mathrm{TB}$ as a barrier for patients in accessing the Rural Health Units (RHU) for diagnosis and treatment. Also, the lack of proper orientation of a City or Provincial Health Office personnel regarding the interventions under PDI in some areas was identified as a problem. A representative from IMPACT resolved this issue through reorientation of the NTP Coordinators to the program.

There were variations in the monitoring process of the technical assistance for each implementation site. Some implementations sites have IMPACT as the main monitoring agency that checks the referral slips from the NTP Coordinators. Other sites name the PDI Project Team as the representative who checks the logbooks of the health centers to confirm if the patients who were referred by the engaged pharmacies accessed the health centers. However, there were implementation sites which reported no monitoring and evaluation conducted in their area throughout the PDI implementation period. Moreover, the lack of feedback at various stages of the referral contributed to the inability to sustain the referrals made by the engaged pharmacies. The reports submitted to the NTP Coordinators by the Area Field Coordinators were limited to the number of engaged pharmacies and trained pharmacy personnel. NTP Coordinators are unable to confirm if the referrals made by each engaged pharmacy where able to reach the RHUs.

\section{DISCUSSION}

This paper aimed to identify the facilitators and barriers to the implementation of PDI in selected project sites. Facilitators were (a) acceptance and cooperation of stakeholders; (b) capability-building of pharmacy personnel; and (c) establishment of a referral system. On the other hand, hindering factors identified can be grouped into patient-related, pharmacy-related, health facility-related, and program-related factors.

Facilitators to implementation of PDI can be conceptualized as being related to stakeholder engagement and participation, which has been recognized in the management literature as an important contributor to project success. ${ }^{10,11}$ This appeared to be crucial in bringing pharmacies - which principally belong to the private sector - to interface with the local TB control program, which is managed by government. Existing relationships between stakeholder groups at the community level enabled the process of obtaining buy-in and commitment, while training of pharmacy owners and pharmacists can be thought of as

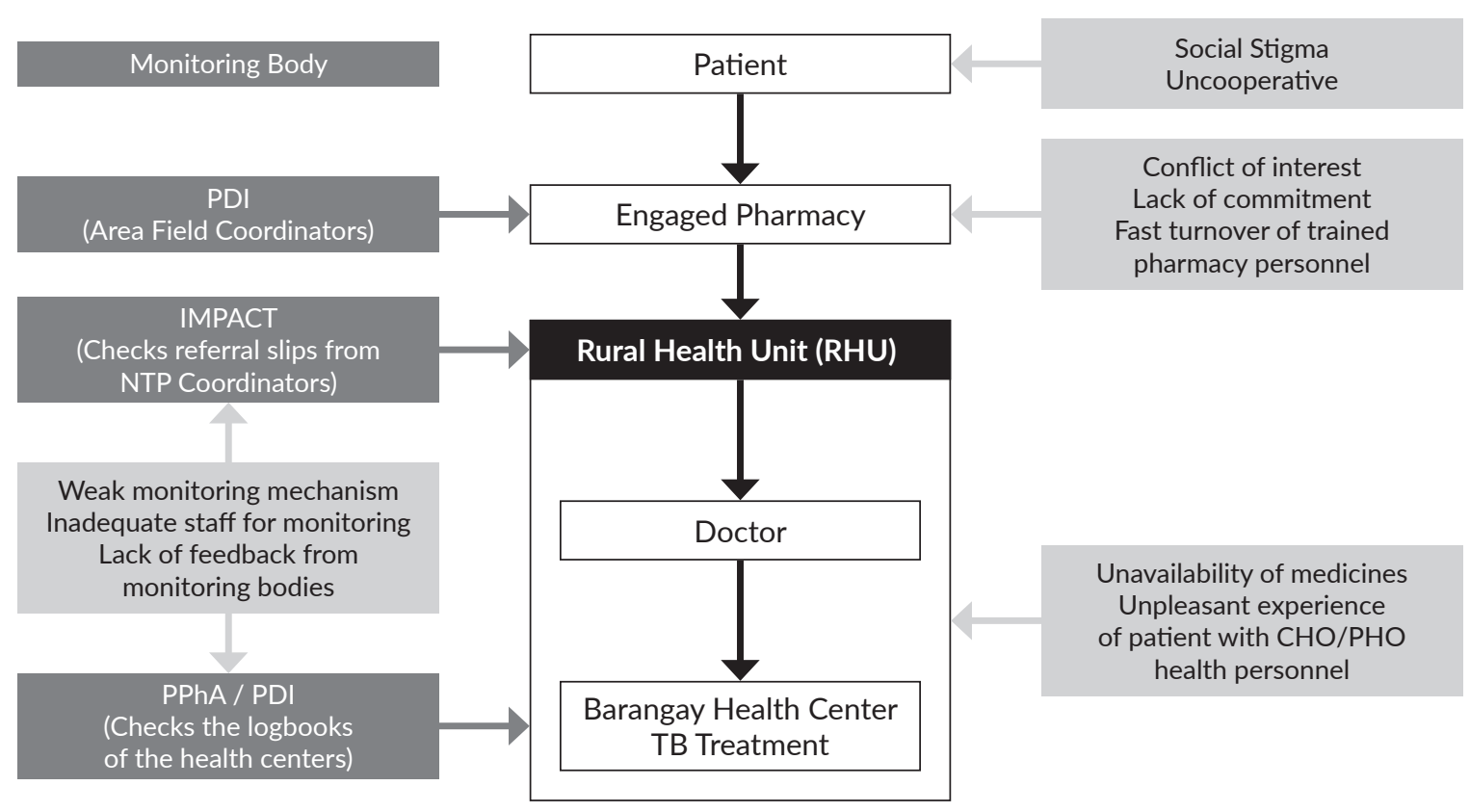

Figure 2. Summary of the hindering factors identified based on the referral system and the monitoring bodies involved in each level. 
Factors Affecting Pharmacy DOTS Initiative

providing scaffolding that strengthened participation in the project. The referral system, on the other hand, reinforced the established bonds among stakeholders as it served as a touchpoint between groups.

Social stigma remains a hindering factor even if interventions addressing TB has been implemented several decades ago. Since TB has a history of social stigma in most countries, most patients consider this as a major factor for not going to health centers when a pharmacy refers them for consultation. ${ }^{12}$ Some of the engaged pharmacies still prioritize business and sales; hence, they continue to dispense the medicines instead of making referrals to health care facilities. Trained pharmacy personnel lack commitment in making referrals and training fellow personnel. The fast turnover of trained pharmacy personnel is also a big hindering factor since the PDI protocol and referrals are not properly endorsed to incoming pharmacy personnel. Inability to commit to the active referral activity contributes to low detection rate of TB in susceptible patients. ${ }^{13}$

If the health center units are unable to provide the TB drugs, then the goal of PDI will not be met for confirmed TB cases. An unstable supply of TB drugs leads to interruptions during the treatment. Patients who experience relief of symptoms and do not receive the medications on time might not recognize the need for continuing the therapy. ${ }^{9}$ Unfriendly or unwelcoming staff in the health center units were also identified to contribute to the refusal of referred patients to return to the health center. The quality of interaction a patient experiences with a health professional or worker affects their decision on receiving and finishing treatment from a DOTS facility. ${ }^{14}$ As for the program-related factors, there were gaps in the monitoring and feedback system mainly attributed to insufficient manpower of the program. The absence of a feedback system in the different levels of the referral system contributed to the loss of interest of engaged pharmacies from continuing with the PDI interventions. Some factors external to the PDI program also hindered its implementation. Pharmacies within the implementation sites but not included in the PDI program continued to dispense anti-TB drugs without prescription. This discouraged patients from going to health centers after receiving the referral, as well as engaged pharmacies from continuing with the referrals. The strong LGU TB advocacy and program in some implementation areas made it difficult to continue with the referrals.

Notably, more hindering factors than facilitating factors were identified from semi-structured interviews with field implementers and the program manager for PDI, which may partly explain the non-attainment of some objectives of $\mathrm{PDI}$, and the difficulty in sustaining the intervention over the project lifetime. ${ }^{7,8}$

Possible measures to address the hindering factors identified in this analysis include the institution of reinforcement measures to address pharmacy-related factors (e.g., reward mechanism, integration in licensing requirements, provision of manuals of procedures), implementing sustained capability-building measures and improving the supply chain management system to address health center-related concerns, and monitoring and feedback mechanisms for program-related factors.

Results presented in this paper will need to be assessed in light of potential limitations to the evaluation conducted. First, only a limited set of project implementation sites were selected for inclusion in the analysis due to feasibility constraints, and one potential respondent declined to participate and was not replaced. While representation in terms of the geographic segmentation used by IMPACT was assured, it is possible that the theoretical universe of possible pharmacy-respondents was limited by the fact that some of the initially-engaged pharmacies were already out of business at the time of field work. ${ }^{8}$ Second, data collection was done at the terminal phase of the implementation of IMPACT, which may have resulted to respondents recalling only parts of facilitators and barriers - most likely the recent ones they have experienced - that may have arisen in the course of project implementation from 2014 to 2016. However, this latter limitation in terms of information may have been partially addressed by the team's inclusion of several respondents who belong to two different groups (i.e., NTP coordinators and the PDI program manager).

\section{CONCLUSION}

The study revealed that PDI was able to engage stakeholders through the capability-building activities implemented and in establishing a referral system through public and private partnerships. However, significant issues were present regarding the sustainability of the program's interventions. The hindering factors involved and affected the patients, pharmacies, health centers, and the PDI program itself. Nonetheless, the PDI was instrumental in the establishment of a referral system and linked the public and private sector for an advocacy against $\mathrm{TB}$.

\section{Acknowledgments}

The authors would like to acknowledge the support and assistance of the National Tuberculosis Control Program (NTP) in the field data collection activities conducted as part of the evaluation. Assistance in preparing this manuscript was provided by Kristine S. Garcia (College of Pharmacy, University of the Philippines Manila).

\section{Statement of Authorship}

$\mathrm{CTA}, \mathrm{ACB}, \mathrm{JPG}$, and KLC conceptualized the project, with guidance from ECM and RMF.JPG developed the data collection instrument. ADA, MDA and JAM participated in the data collection. JPG, DAO, and CTA analyzed the data. DAO and JPG prepared the first draft of the manuscript. JPG finalized the manuscript based on comments from other authors. All other authors reviewed results and/or 
contributed to the report. All authors approved the final version submitted.

\section{Author Disclosure}

CTA, ACB and KLC received professional fees and grants from Johnson \& Johnson (Philippines), Inc., outside of the submitted work. All other authors declare no potential conflicts of interest with respect to the research, authorship, and/or publication of this article.

\section{Funding Source}

This technical paper is made possible by the generous support of the American people through the United States Agency for International Development (USAID) under Cooperative Agreement No. AID-492-A-12-00014 implemented by the Philippine Business for Social Progress.

\section{REFERENCES}

1. World Health Organization. Global Tuberculosis Report 2016. Geneva: World Health Organization; 2016.

2. Vianzon R, Garfin A, Lagos A, Belen R. The tuberculosis profile of the Philippines, 2003-2011: Advancing DOTS and beyond. Western Pacific Surveillance and Response. 2013;4(2):11-6. doi:10.5365/ wpsar.2012.3.4.022.

3. Department of Health. Second National Drug Resistance Survey on Tuberculosis in the Philippines. Manila: Department of Health; 2014.

4. Department of Health. 2010-2016 Philippine Plan of Action to Control Tuberculosis (DOH HSRA Monograph No. 11). Manila: Department of Health; 2010.

5. Lönnroth K, Karlsson M, Lan NT, Buu TN, Dieu TT. Referring TB suspects from private pharmacies to the National Tuberculosis Programme: Experiences from two districts in Ho Chi Minh city, Vietnam. Int J Tuberc Lung Dis. 2003;7(12):1147-53.
6. USAID. Innovations and Multi-Sectoral Partnerships to Achieve Control of TB (IMPACT) [Internet]. 2016 [cited 2017 Feb 21]. Available from: https://www.usaid.gov/philippines/health/impact.

7. Cochon KL, Bermudez AC, Orolfo DA, Guevarra JP, Avelino $\mathrm{MD}$, Agbon AG, et al. Outputs and outcome of the Pharmacy Directly-Observed Treatment Short-Course (DOTS) initiative in the Philippines. Phil J Health Res Dev. 2019;23(1):48-53.

8. Bermudez AC, Antonio CT, Guevarra JP, Cochon KL, Orolfo DA, Manalo JA, et al. Pharmacy DOTS Initiative (PDI): A case study on integrating pharmacies in the tuberculosis directly observed treatment-short course (TB DOTS) network in the Philippines. Phil J Health Res Dev. 2020;24(1):59-64.

9. Nowell LS, Norris JM, White DE, Moules NJ. Thematic analysis: Striving to meet the trustworthiness criteria. Int J Qual Methods. 2017;16:1-13. doi: 10.1177/1609406917733847.

10. Assudani R, Kloppenborg TJ. Managing stakeholders for project management success: An emergent model of stakeholders. J Gen Manag. 2010;35(3):67-80. doi: 10.1177/030630701003500305

11. Retfalvi LA. Project success using proven stakeholder management techniques. Paper presented at PMI ${ }^{\circledR}$ Global Congress 2014North America, Phoenix, AZ. Newtown Square, PA: Project Management Institute.

12. Diefenbach-Elstob T, Plummer D, Dowi R, Wamagi S, Gula B, Siwaeya K, et al. The social determinants of tuberculosis treatment adherence in a remote region of Papua New Guinea. BMC Public Health. 2017;17:70. doi: 10.1186/s12889-016-3935-7.

13. Bell CA, Ilomäki J, Pichenda K, Duncan GJ, Saini B. Referral of tuberculosis symptomatic clients from private pharmacies to public sector clinics for diagnosis and treatment in Cambodia. J Eval Clin Prac. 2015;21(2):285-91. doi: 10.1111/jep.12306.

14. Gebremariam MK, Bjune GA, Frich JC. Barriers and facilitators of adherence to TB treatment in patients on concomitant TB and HIV treatment: A qualitative study. BMC Public Health. 2010;10:651. doi: $10.1186 / 1471-2458-10-651$. 